\title{
Experimental Engineering of Arbitrary Qudit States with Discrete-Time Quantum Walks
}

\author{
Taira Giordani, ${ }^{1}$ Emanuele Polino, ${ }^{1}$ Sabrina Emiliani, ${ }^{1}$ Alessia Suprano, ${ }^{1}$ Luca Innocenti, ${ }^{2}$ Helena Majury, ${ }^{2}$ \\ Lorenzo Marrucci, ${ }^{3}$ Mauro Paternostro, ${ }^{2}$ Alessandro Ferraro, ${ }^{2}$ Nicolò Spagnolo, ${ }^{1}$ and Fabio Sciarrino ${ }^{1,4}$ \\ ${ }^{1}$ Dipartimento di Fisica, Sapienza Università di Roma, Piazzale Aldo Moro 5, I-00185 Roma, Italy \\ ${ }^{2}$ Centre for Theoretical Atomic, Molecular, and Optical Physics, School of Mathematics and Physics, \\ Queen's University Belfast, BT7 INN Belfast, United Kingdom \\ ${ }^{3}$ Dipartimento di Fisica "Ettore Pancini”, Università Federico II, Complesso Universitario di Monte Sant’Angelo, \\ Via Cintia, 80126 Napoli, Italy \\ ${ }^{4}$ Consiglio Nazionale delle Ricerche, Istituto dei sistemi Complessi (CNR-ISC), \\ Via dei Taurini 19, 00185 Roma, Italy
}

(Received 3 August 2018; published 18 January 2019)

\begin{abstract}
The capability to generate and manipulate quantum states in high-dimensional Hilbert spaces is a crucial step for the development of quantum technologies, from quantum communication to quantum computation. One-dimensional quantum walk dynamics represents a valid tool in the task of engineering arbitrary quantum states. Here we affirm such potential in a linear-optics platform that realizes discrete-time quantum walks in the orbital angular momentum degree of freedom of photons. Different classes of relevant qudit states in a six-dimensional space are prepared and measured, confirming the feasibility of the protocol. Our results represent a further investigation of quantum walk dynamics in photonics platforms, paving the way for the use of such a quantum state-engineering toolbox for a large range of applications.
\end{abstract}

DOI: 10.1103/PhysRevLett.122.020503

Introduction.-The preparation of high-dimensional quantum states is of great significance in quantum information science and technology. Compared to qubits, qudit states-describing quantum systems in $d$-dimensional spaces-enable stronger foundational tests of quantum mechanics [1-3] and better-performing applications in secure quantum communications [4-9], quantum emulation [10,11], quantum error correction [12-14], faulttolerant quantum computation [15-19], and quantum machine learning [20-22].

Protocols performed on systems living in large Hilbert spaces require great control in light of the number of parameters required to describe states and operations. Nonetheless, qudit states have been prepared successfully in various physical settings [11,23-32]. Such schemes rely on ad hoc strategies whose dependence on the underpinning dynamics makes their translation across different physical platforms difficult.

A promising way to achieve a higher degree of platform universality is the use of the rich dynamics offered by quantum walks (QWs) [33-35]. These can be thought of as the quantum counterparts of classical random walks and comprise - in their discrete version-a qudit, named walker, endowed with an internal two-dimensional degree of freedom dubbed coin [36]. QWs have been successfully implemented [37] in systems as diverse as trapped atoms [38] and ions [39,40], photonic circuits [41-50], and optical lattices [51]. An approach for state engineering based on their dynamics offers hope of being applicable in a variety of different systems, independently of the physical implementation.

While the QW dynamics was previously shown to allow the engineering of specific walker's states [52,53], in Ref. [54] a scheme was proposed to use discrete-time QWs on a line to prepare arbitrary qudit states with high probability and fidelity, in principle for arbitrary dimensions of target qudit states. This is achieved by enhancing the degree of control over walk's dynamics through the arrangement of suitable step-dependent coin operations, which affect the coin-walker quantum correlations by de facto steering the state of the walker towards the desired final state, and finally projecting in the coin space. This removes correlations between walker and coin, thus producing a pure walker state with the desired features.

In this Letter, we use the scheme of Ref. [54] to demonstrate a state-engineering protocol based on the controlled dynamics generated by QWs. We use the orbital angular momentum (OAM) degree of freedom of singlephoton states as a convenient embodiment of the walker $[48,55,56]$. OAM-based experiments offer the possibility to cover Hilbert spaces of large dimensions in light of the favorable (linear) scaling of the number of optical elements with the size of the walk. Moreover, the scheme allows for full control of the coin operation that is key to the walk implementation. In order to demonstrate the versatility of our scheme, we focus on the interesting classes of large superpositions of OAM states [57,58]. Furthermore, we show experimentally the capability of engineering arbitrary 
states. The quality of the generated states and the feasibility of the experimental protocol that we have put in place, demonstrate the effectiveness of a hybrid platform for quantum state engineering. Such platform holds together a programmable quantum system, the photonic QW in the angular momentum, and classical optimization algorithms to effectively reach a given target.

Engineering quantum walks.-We consider a discretetime QW with a two-dimensional coin with logical states labeled as $\left\{|\downarrow\rangle_{c},|\uparrow\rangle_{c}\right\}$. The dynamics are made up of consecutive unitary steps. At step $t$, a coin operator $\hat{\mathcal{C}}_{t}$ changes the coin state and is then followed by a shift operator $\hat{\mathcal{S}}_{w c}$, which moves the walker conditionally to the coin state. Such transformations are described by the operators

$$
\hat{\mathcal{C}}_{t}=\left(\begin{array}{ll}
e^{i \xi_{t}} \cos \theta_{t} & e^{i \zeta_{t}} \sin \theta_{t} \\
-e^{-i \zeta_{t}} \sin \theta_{t} & e^{-i \xi_{t}} \cos \theta_{t}
\end{array}\right),
$$

which accounts for coin tossing, and $\hat{\mathcal{S}}_{w c}=\sum_{k}|k-1\rangle$ $\left\langle\left. k\right|_{w} \otimes \mid \downarrow\right\rangle\left\langle\left.\downarrow\right|_{c}+\mid k+1\right\rangle\left\langle\left. k\right|_{w} \otimes \mid \uparrow\right\rangle\left\langle\left.\uparrow\right|_{c}\right.$, which realizes the conditional motion of the walker. Here $k$ is the lattice-site occupied by the walker and $\left\{\theta_{t}, \xi_{t}, \zeta_{t}\right\}$ are parameters identifying a unitary transformation in two dimensions. The evolution through $n$ steps of the QW is given by $\hat{U}=\prod_{t=1}^{n} \hat{\mathcal{S}}_{w c} \hat{\mathcal{C}}_{t}$.

In Ref. [54] it was shown that it is always possible to find a set of coin operators $\left\{\hat{\mathcal{C}}_{t}\right\}_{t=1}^{n}$ that produce an arbitrary target state in the full coin-walker space. In addition, via suitable projection in the coin space, arbitrary walker states can also be obtained. The identification of the correct set of coin operators is enabled by a classical algorithm to maximize the fidelity between the final state of the walker, after projection of the coin, and the target $(n+1)$-dimensional state. It is worth noting that all states can be reachable with unit fidelities (albeit probabilistically) and high probabilities, regardless of the number of steps $n$ (see Ref. [59]).

To demonstrate the effectiveness of this approach for state engineering of high-dimensional spaces, we here focus on classes of physically relevant states. First, we consider the synthesis of superposition of large OAM states (SLOS) achieved by engineering coherent superpositions of extremal walker positions [62]. To this end, we exploit the correspondence between the position space of the walker and an angular momentum of quantum number $n / 2$. These states play a crucial role in the investigations on foundations of quantum mechanics [63] and their generation is at the core of various quantum engineering protocols $[57,58,64,65]$. The second class of states that we consider is spin-coherent states [66], which are the spinlike counterpart of coherent states of a quantum harmonic oscillator. Finally, in order to validate the flexibility of our approach, we demonstrate high-quality engineering of both balanced and randomly sampled states.
Experimental apparatus. - We have implemented a discrete-time QW with $n=5$ steps, using the angular momentum states of light $\left\{|m\rangle_{w}\right\} \quad(m= \pm 5, \pm 3, \pm 1)$ as the physical embodiment of the walker, while logical states of the coin are encoded in circular-polarization states $\{|R\rangle,|L\rangle\}$. We dub such degree of freedom as spin angular momentum (SAM) to mark the difference with OAM. Our experimental setup, which is shown schematically in Fig. 1 and follows Refs. [48,49], allows for the full coin-walk evolution to take place in a single light beam, thus avoiding a nonlinear growth of optical paths as in previous interferometric implementations $[48,55,56]$. Such a scheme guarantees a linear scaling of the number of optical elements needed to implement a $n$-step QW (see Ref. [59]). Arbitrary coin operators are achieved through a sequence of suitably arranged and oriented quarter- and half-wave plates [67]. The shift operator $\hat{\mathcal{S}}_{w c}$ is instead implemented using a $Q$ plate (QP) [68], an active device that uses an inhomogeneous birefringent medium to convert SAM into OAM and that can conditionally change the values of the OAM by a quantity $2 q$ (here $q$ is the topological charge of the device) according to transformations

$$
\begin{aligned}
& |L, m\rangle \stackrel{\mathrm{QP}}{\longrightarrow} \cos \frac{\delta}{2}|L, m\rangle+i e^{2 i \alpha_{0}} \sin \frac{\delta}{2}|R, m+2 q\rangle, \\
& |R, m\rangle \stackrel{\mathrm{QP}}{\longrightarrow} \cos \frac{\delta}{2}|R, m\rangle+i e^{-2 i \alpha_{0}} \sin \frac{\delta}{2}|L, m-2 q\rangle .
\end{aligned}
$$

The additional phase $\alpha_{0}$ between the two polarizations is compensated by changing the orientations of the wave plates which implement the coin operator of the subsequent step.

Single-photon states are generated via a type-II, collinear spontaneous-parametric-down-conversion source [cf. Fig. 1]. The photons emitted by the source are separated with a polarizing beam splitter (PBS) and coupled to two single-mode fibers (SMF). One photon acts as the trigger signal, while the other one undergoes the QW evolution. After propagation in the SMF and the first PBS, the initial state of the walker and coin is prepared in $\left|\psi_{0}\right\rangle_{w c}=|0\rangle_{w} \otimes|+\rangle_{c}$ with $|+\rangle_{c}=\left(|\uparrow\rangle_{c}+|\downarrow\rangle_{c}\right) /$ $\sqrt{2}$. At the end of an $n$-step QW, the protocol involves a projection of the coin state onto $|+\rangle_{c}$. This is experimentally implemented by a final PBS. The OAM analysis is performed through a spatial-light modulator (SLM) followed by coupling into a single-mode fiber, which allows for the measurement of arbitrary superposition of OAM components with high accuracy [69,70]. The quantum state fidelity between the actual state of the walker and the target $(n+1)$-dimensional state is estimated by projecting the OAM state onto a basis that contains the given target state [cf. Fig. 1].

Engineering superposition of large OAM states.-Our investigation on the engineering of quantum states living in Hilbert spaces of large dimensions starts from coherent superpositions of two extremal lattice sites of the walker. 
(a)

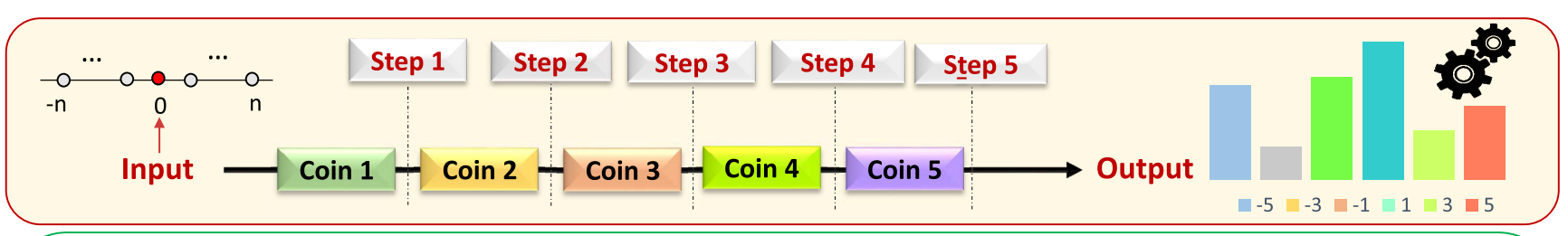

(b)

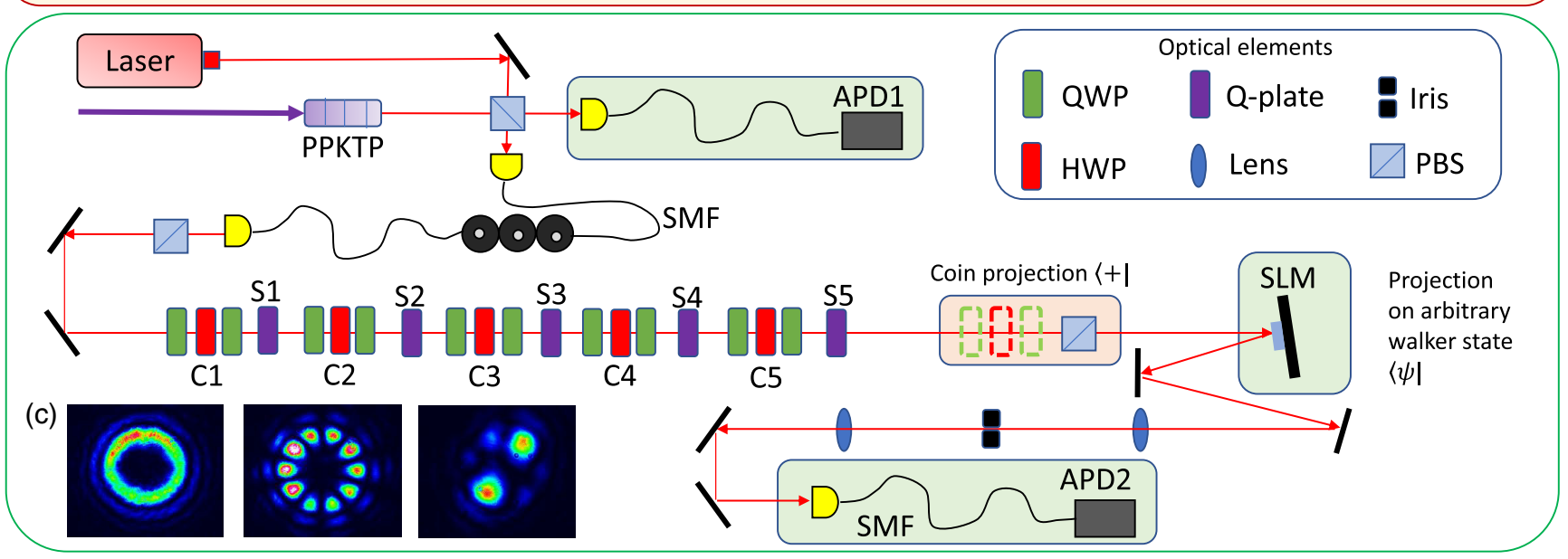

FIG. 1. Setup for the quantum state engineering toolbox. (a) Conceptual scheme of the protocol. At each step of the QW the coin operator is changed to obtain a target state in the output. (b) A single-photon source, composed of a periodically poled potassium titanyl phosphate (PPKTP) crystal, generates pairs of photons that are coupled in a single-mode fiber. One photon acts as trigger while the other is prepared in $\left|\psi_{0}\right\rangle=|+\rangle \otimes|0\rangle$ through polarization controllers and a polarizing beam splitter. Five sets of quarter (QWP) and half (HWP) wave plates implement operators $\left\{C_{i}\right\}$ for each step. Five $Q$ plates implement the shift operator of the $\mathrm{QW}\left\{S_{i}\right\}$. The detection stage consists of a PBS followed by a spatial light modulator, a SMF and an avalanche photodiode detector (APD), for projection onto $|+\rangle \otimes|\psi\rangle$. (c) Pictures of OAM modes of the output states after PBS, obtained with coherent light. From right: OAM eigenstate corresponding to $m=5$; balanced superposition of $m= \pm 5$; balanced superposition of all OAM components covered by the five-step QW $m=\{ \pm 5, \pm 3, \pm 1\}$.

The isomorphism of the OAM with an angular momentum of quantum number $n / 2$ allows us to put in correspondence position states of the walker on the lattice $| \pm 5\rangle$ with angular momentum states with minimum and maximum projections onto the quantization axis $| \pm 5 / 2\rangle$ (for simplicity of notation, we will use position states only). Hence, the superposition state $\left(|5\rangle+e^{i \varphi}|-5\rangle\right) / \sqrt{2}$ (with $\varphi$ a suitable phase) allows us to benchmark the performance of our experiment with a relevant class of states $[52,53,65]$ used in quantum sensing [71,72].

In Fig. 2 we report experimental results for the generation of four of such states, which are conveniently pictured as states pointing towards the poles of a Blochlike ball. Quantum coherence between the components of such states has been tested by changing their relative phase. The values of the state fidelity between experimentally synthesized states and their respective target ones are reported in Fig. 2. Hereafter we compute fidelities by projecting the state on the orthonormal basis which includes the target qudit in the six-dimensional subspace associated to our five-step QW, generated by OAM eigenstates $\left\{|m\rangle_{w}\right\}(m= \pm 5, \pm 3, \pm 1)$.

The second class of relevant states that we addressed are spin-coherent states (SCSs) [64]. These are the counterparts of harmonic oscillator coherent states for a particle with spin $s$ [64,73-75]. SCSs are eigenstates-with eigenvalue $s$-of the component of the total spin-momentum operator $\hat{S}$ pointing along the direction identified by the polar spherical angles $\{\theta, \phi\}[64,66,74,76]$. A decomposition of such states over the $\left\{\left|s_{z}\right\rangle\right\}$ basis of the projected spin along the $z$ direction $\left(\hat{S}_{z}\right)$ reads

$$
|s, \theta, \phi\rangle=\sum_{s_{z}=-s}^{s} \sqrt{\frac{(2 s) !}{\left(s+s_{z}\right) !\left(s-s_{z}\right) !}} e^{-i \phi s_{z}} C_{\theta}^{s+s_{z}} S_{\theta}^{S-s_{z}}\left|s_{z}\right\rangle,
$$

with $C_{\theta}=\sqrt{1-S_{\theta}^{2}}=\cos (\theta / 2)$. SCSs have various applications in condensed matter physics, in particular in quasiexactly solvable models, for Wigner-Kirkwood expansion and in quantum correction to energy quantization rules [66]. At the foundational level, they can be used to generate Schrödinger cat states [64].

Although SCSs are in general not orthogonal, they form a convenient basis. Moreover, as two SCSs pointing in opposite azimuthal directions are orthogonal for $\theta \sim \pi / 2$, by restricting the attention to $\{|s, \pi / 2, \phi\rangle,|s,-\pi / 2, \phi\rangle\}$ we would be dealing with an orthonormal basis, which we 
(a)

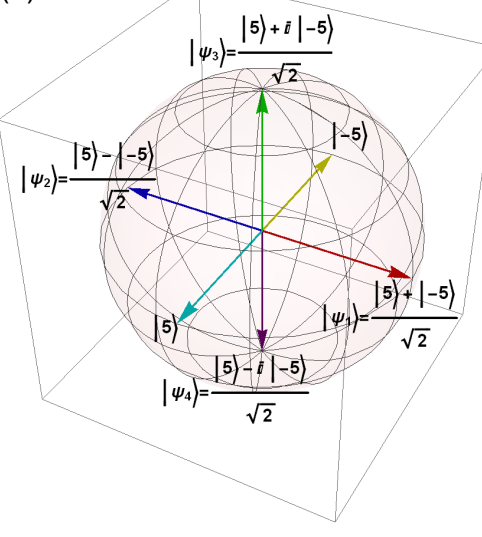

(b)

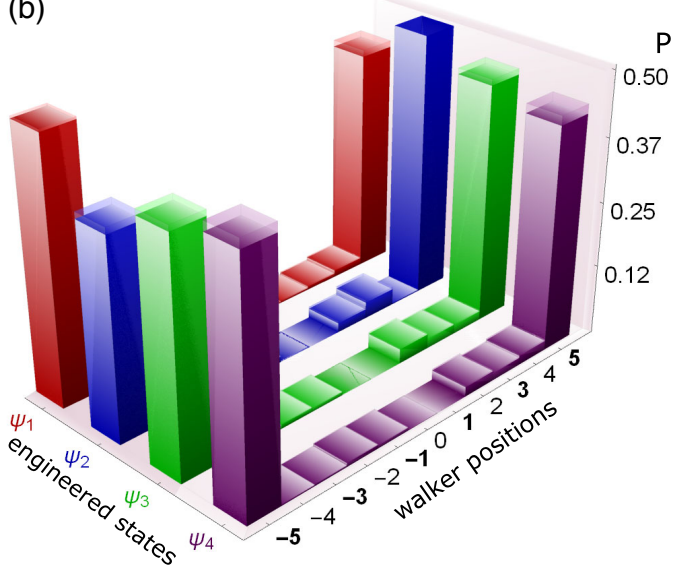

(c)

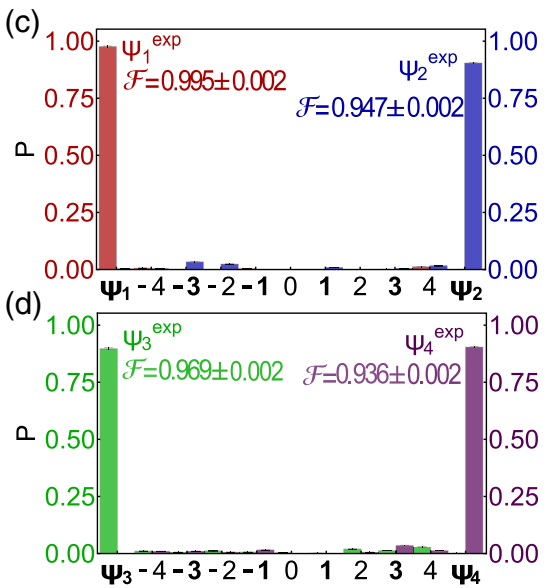

FIG. 2. Experimental results for the engineering of SLOS states. (a) Representation on a Bloch-like ball of the four target states corresponding to the superposition of $| \pm 5\rangle$, corresponding to OAM states with maximum and minimum projection of the angular momentum along the quantization axis. (b) Population of the OAM components after five-step QW for states $\left|\psi_{i}\right\rangle(i=1,2,3,4)$ in panel (a). Odd- $m$ position states (bold numbers on the $x$ axis) should be the only ones involved in the state engineering. However, we report also the populations of even- $m$ position states (light-black numbers on the $x$ axis) to illustrate possible imperfections at generation and detection stages. The error bars associated with experimental populations are shown by the transparent areas on top of each histogram. (c)-(d) Distributions of probabilities $P_{i}=\left\langle B_{i}^{(j)}\left|\rho_{\exp }\right| B_{i}^{(j)}\right\rangle(j=1,2)$ that the experimental walker state $\rho_{\exp }$ is found to be one of the elements of the bases $B^{(j)}=\left\{\left|\psi_{p}\right\rangle,\left|\psi_{p+1}\right\rangle,| \pm 4\rangle,| \pm 3\rangle,| \pm 2\rangle,| \pm 1\rangle,|0\rangle\right\}$ with $p=1$ for $j=1$ and $p=3$ for $j=2$. All the error bars are due to Poissonian uncertainties, propagated through Monte Carlo methods.

can use to construct the analogous of a Bloch ball for a two-level system [cf. Fig. 3(a)]. We have thus engineered $\left|S_{1}\right\rangle \equiv|5 / 2, \pi / 2,0\rangle$ and $\left|S_{2}\right\rangle \equiv|5 / 2,-\pi / 2,0\rangle$, and considered the experimental synthesis of balanced coherent superpositions of such states. Furthermore, $S_{1}$ and $S_{2}$ are also eigenstates of $\hat{S}_{x}$ operator. In Figs. 3(b)-3(c) the state $S_{1}$ is projected firstly on the computational basis, the eigenstates of $\hat{S}_{z}$, and then on the basis $\left\{S_{i}\right\}$, with $i=1, \ldots, 6$, which consists of $\hat{S}_{x}$ eigenstates. Balanced superpositions of $S_{1}$ and $S_{2}$ are akin to superposition of coherent states of a harmonic oscillator, as they exhibit signatures of nonclassical interference [59,64]. For instance, only even (odd) components of the logical basis enter the superposition $\left|S_{1}\right\rangle+\left|S_{2}\right\rangle\left(\left|S_{1}\right\rangle-\left|S_{2}\right\rangle\right)$. Hence, we can generate SCS mapping the basis $\left\{\left|s_{z}\right\rangle\right\}$ in Eq. (3) into the basis of the QW $\left\{|m\rangle_{w}\right\}$. In Figs. 3(a)-3(e) we show the high quality of the generated states.

Engineering arbitrary qudits.-In order to demonstrate the flexibility of our scheme, we have addressed the generation of states of arbitrary complexity, starting from (a)

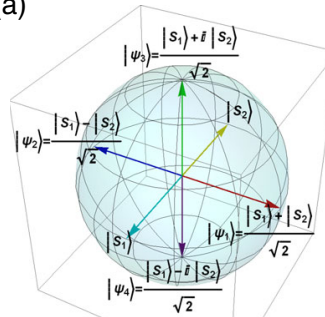

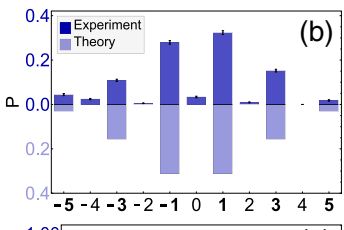

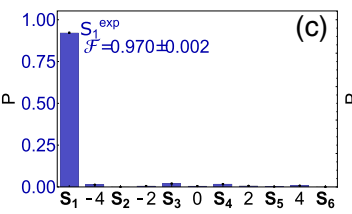

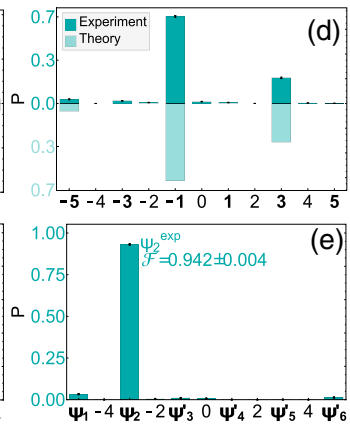

(d)

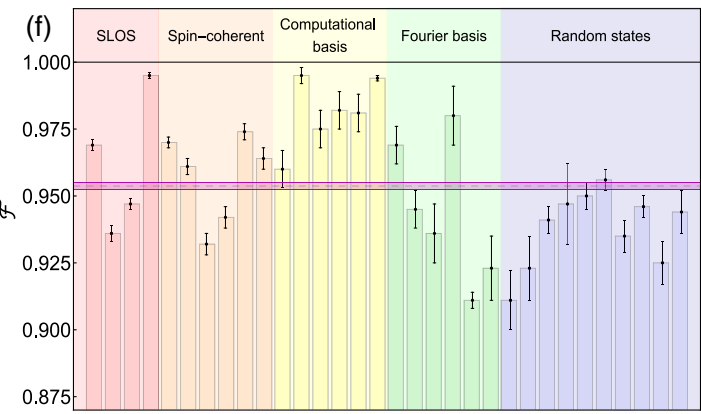

FIG. 3. Experimental results for the engineering of SCSs and their coherent superposition: (a) Bloch-sphere representation for the mutually orthogonal SCSs $\left|S_{1}\right\rangle$ and $\left|S_{2}\right\rangle$. (b) Probability distributions associated to the projection of $\left|S_{1}\right\rangle$ onto the computational basis. As previously explained, we also consider the contribution of even OAM components. (c) Probability distribution corresponding to the basis that contains the target state itself $\left|S_{1}\right\rangle$, generated with the fidelity reported in the panel. Such orthonormal basis, $S_{i}$ with $i=1, \ldots, 6$, contains eigenstates of $\hat{S}_{x}$ for a particle with spin $s=5 / 2$ that are in turn all spin-coherent states. (d) Experimental probability distribution on computational basis for $\left|\psi_{2}\right\rangle=(1 / \sqrt{2})\left(\left|S_{1}\right\rangle-\left|S_{2}\right\rangle\right)$. Only components $\{-5,-1,3\}$, corresponding to logical states $\{1,3,5\}$, have nonzero probabilities. (e) Quantum state fidelity evaluated measuring state $\left|\psi_{2}\right\rangle$ on the orthonormal basis that contains state $\left|\psi_{1}\right\rangle$, as described in the main text. (f) Summary of quantum state fidelities for the 32 states generated in the experiment. Magenta area: average fidelity $\overline{\mathcal{F}}=0.954 \pm 0.001$. 
balanced states and then moving towards randomly chosen states. Balanced states are challenging as one needs to ensure equal population of all their components, a condition that is very prone to experimental imperfections. Assessing the quality of generation of such states provides a significant benchmark to the effectiveness of the procedure. We have then engineered the element of a Fourier basis associated to the Hilbert space of the walker. This choice is motivated by the importance of quantum Fourier transform in quantum algorithms [77], as well as its role in identifying mutually unbiased bases for quantum cryptography and communication in high-dimensions [70,78-80].

Final measurements concern the generation of randomly chosen qudits. We have engineered up to 5 states with realvalued amplitudes and 5 with complex-valued ones, where the state components are sampled from a uniform distribution (cf. Ref. [59]). In Fig. 3(f) quantum state fidelities are reported for all experimental engineered states, including the Fourier basis and randomly sampled qudits, where the magenta area shows the average fidelity and its uncertainty $(\overline{\mathcal{F}}=0.954 \pm 0.001)$ [59]. Such a test provides a further proof of the effectiveness of the strategy demonstrated in our experiment.

Discussion.-We have successfully tested a QW-based quantum state engineering strategy assisted by numerical optimization [54]. Our tests were performed in a photonic platform using OAM as the embodiment of a quantum walker. This allowed us to implement a five-step QW, without nonlinear overhead in the number of required optical paths and with full control on the preparation, coin-operation, and detection stages. We showed the effectiveness of the protocol, demonstrating its ability to synthesize high-quality qudit states. Our results reinforce the idea that numerical optimization complementing a complex QW dynamics is effective for high-dimensional state engineering. A natural generalization of this novel paradigm could be the engineering in the multipartite scenario, exploiting quantum correlations between multiple walkers. Regarding the research of the coin, further improvements of our approach can be envisaged by identifying appropriate routines to optimize the state engineering process in the presence of actual experimental imperfections. To this end, machine learning algorithms can be a promising add-on to our numerical optimization approach to adapt the coin operators to a given experimental implementation.

We acknowledge support from the ERC-Advanced Grant PHOSPhOR (Photonics of Spin-Orbit Optical Phenomena; Grant Agreement No. 694683), the Northern Ireland Department for Economy (NI DfE), the DfE-SFI Investigator Programme (Grant No. 15/IA/ 2864), and the H2020 Collaborative Project TEQ (Grant No. 766900).
[1] T. Vértesi, S. Pironio, and N. Brunner, Phys. Rev. Lett. 104, 060401 (2010).

[2] N. Brunner, D. Cavalcanti, S. Pironio, V. Scarani, and S. Wehner, Rev. Mod. Phys. 86, 419 (2014).

[3] R. Lapkiewicz, P. Li, C. Schaeff, N. K. Langford, S. Ramelow, M. Wieśniak, and A. Zeilinger, Nature (London) 474, 490 (2011).

[4] H. Bechmann-Pasquinucci and A. Peres, Phys. Rev. Lett. 85, 3313 (2000).

[5] M. Fitzi, N. Gisin, and U. Maurer, Phys. Rev. Lett. 87, 217901 (2001)

[6] N. J. Cerf, M. Bourennane, A. Karlsson, and N. Gisin, Phys. Rev. Lett. 88, 127902 (2002).

[7] D. Bruß and C. Macchiavello, Phys. Rev. Lett. 88, 127901 (2002).

[8] A. Acin, N. Gisin, and V. Scarani, Quantum Inf. Comput. 3, 563 (2003).

[9] N. K. Langford, R. B. Dalton, M. D. Harvey, J. L. O’Brien, G. J. Pryde, A. Gilchrist, S. D. Bartlett, and A. G. White, Phys. Rev. Lett. 93, 053601 (2004).

[10] I. Buluta and F. Nori, Science 326, 108 (2009).

[11] M. Neeley, M. Ansmann, R. C. Bialczak, M. Hofheinz, E. Lucero, A. D. O'Connell, D. Sank, H. Wang, J. Wenner, A. N. Cleland, M. R. Geller, and J. M. Martinis, Science 325, 722 (2009).

[12] I. L. Chuang, D. W. Leung, and Y. Yamamoto, Phys. Rev. A 56, 1114 (1997).

[13] G. Duclos-Cianci and D. Poulin, Phys. Rev. A 87, 062338 (2013).

[14] M. H. Michael, M. Silveri, R. T. Brierley, V. V. Albert, J. Salmilehto, L. Jiang, and S. M. Girvin, Phys. Rev. X 6, 031006 (2016).

[15] S. D. Bartlett, H. de Guise, and B. C. Sanders, Phys. Rev. A 65, 052316 (2002).

[16] T. C. Ralph, K. J. Resch, and A. Gilchrist, Phys. Rev. A 75, 022313 (2007).

[17] B. P. Lanyon, M. Barbieri, M. P. Almeida, T. Jennewein, T. C. Ralph, K. J. Resch, G. J. Pryde, J. L. O'Brien, A. Gilchrist, and A. G. White, Nat. Phys. 5, 134 (2009).

[18] E. T. Campbell, H. Anwar, and D. E. Browne, Phys. Rev. X 2, 041021 (2012).

[19] E. T. Campbell, Phys. Rev. Lett. 113, 230501 (2014).

[20] M. Schuld, I. Sinayskiy, and F. Petruccione, Contemp. Phys. 56, 172 (2015).

[21] V. Dunjko and H. J. Briegel, arXiv:1709.02779.

[22] J. Biamonte, P. Wittek, N. Pancotti, P. Rebentrost, N. Wiebe, and S. Lloyd, Nature (London) 549, 195 (2017).

[23] D. Leibfried, D. M. Meekhof, B. E. King, C. Monroe, W. M. Itano, and D. J. Wineland, Phys. Rev. Lett. 77, 4281 (1996).

[24] M. Hofheinz, H. Wang, M. Ansmann, R. C. Bialczak, E. Lucero, M. Neeley, A. D. O'Connell, D. Sank, J. Wenner, J. M. Martinis, and A. N. Cleland, Nature (London) 459, 546 (2009).

[25] B. E. Anderson, H. Sosa-Martinez, C. A. Riofrío, I. H. Deutsch, and P. S. Jessen, Phys. Rev. Lett. 114, 240401 (2015).

[26] S. P. Walborn, D. S. Lemelle, M. P. Almeida, and P. H. S. Ribeiro, Phys. Rev. Lett. 96, 090501 (2006). 
[27] G. Lima, L. Neves, R. Guzmán, E. S. Gómez, W. A. T. Nogueira, A. Delgado, A. Vargas, and C. Saavedra, Opt. Express 19, 3542 (2011).

[28] A. Rossi, G. Vallone, A. Chiuri, F. De Martini, and P. Mataloni, Phys. Rev. Lett. 102, 153902 (2009).

[29] A. C. Dada, J. Leach, G. S. Buller, M. J. Padgett, and E. Andersson, Nat. Phys. 7, 677 (2011).

[30] R. W. Heeres, P. Reinhold, N. Ofek, L. Frunzio, L. Jiang, M. H. Devoret, and R. J. Schoelkopf, Nat. Commun. 8, 94 (2017).

[31] S. Rosenblum, Y. Y. Gao, P. Reinhold, C. Wang, C. J. Axline, L. Frunzio, S. M. Girvin, L. Jiang, M. Mirrahimi, M. H. Devoret, and R. J. Schoelkopf, Nat. Commun. 9, 652 (2018).

[32] Y. Chu, P. Kharel, T. Yoon, L. Frunzio, P. T. Rakich, and R. J. Schoelkopf, arXiv:1804.07426.

[33] Y. Aharonov, L. Davidovich, and N. Zagury, Phys. Rev. A 48, 1687 (1993).

[34] J. Kempe, Contemp. Phys. 44, 307 (2003).

[35] S. E. Venegas-Andraca, Quantum Inf. Process. 11, 1015 (2012).

[36] A. Ambainis, E. Bach, A. Nayak, A. Vishwanath, and J. Watrous, in STOC '01 Proceedings of the Thirty-Third Annual ACM Symposium on Theory of Computing (ACM, New York, USA, 2001), pp. 50-59.

[37] K. Manouchehri and J. Wang, Physical Implementation of Quantum Walks (Springer Berlin Heidelberg, Berlin, Heidelberg, 2014).

[38] R. Côté, A. Russell, E. E. Eyler, and P. L. Gould, New J. Phys. 8, 156 (2006).

[39] H. Schmitz, R. Matjeschk, C. Schneider, J. Glueckert, M. Enderlein, T. Huber, and T. Schaetz, Phys. Rev. Lett. 103, 090504 (2009).

[40] F. Zähringer, G. Kirchmair, R. Gerritsma, E. Solano, R. Blatt, and C.F. Roos, Phys. Rev. Lett. 104, 100503 (2010).

[41] H. B. Perets, Y. Lahini, F. Pozzi, M. Sorel, R. Morandotti, and Y. Silberberg, Phys. Rev. Lett. 100, 170506 (2008).

[42] A. Peruzzo, M. Lobino, J. C. F. Matthews, N. Matsuda, A. Politi, K. Poulios, X.-Q. Zhou, Y. Lahini, N. Ismail, K. Wörhoff, Y. Bromberg, Y. Silberberg, M. G. Thompson, and J. L. O'Brien, Science 329, 1500 (2010).

[43] M. A. Broome, A. Fedrizzi, B. P. Lanyon, I. Kassal, A. Aspuru-Guzik, and A. G. White, Phys. Rev. Lett. 104, 153602 (2010).

[44] A. Schreiber, K. N. Cassemiro, V. Potoček, A. Gábris, P. J. Mosley, E. Andersson, I. Jex, and C. Silberhorn, Phys. Rev. Lett. 104, 050502 (2010).

[45] P. P. Rohde, A. Schreiber, M. Štefaňák, I. Jex, and C. Silberhorn, New J. Phys. 13, 013001 (2011).

[46] L. Sansoni, F. Sciarrino, G. Vallone, P. Mataloni, A. Crespi, R. Ramponi, and R. Osellame, Phys. Rev. Lett. 108, 010502 (2012).

[47] J. Boutari, A. Feizpour, S. Barz, C. Di Franco, M. S. Kim, W. S. Kolthammer, and I. A. Walmsley, J. Opt. 18, 094007 (2016).

[48] F. Cardano, F. Massa, H. Qassim, E. Karimi, S. Slussarenko, D. Paparo, C. de Lisio, F. Sciarrino, E. Santamato, R. W. Boyd, and L. Marrucci, Sci. Adv. 1, e1500087 (2015).
[49] F. Cardano, M. Maffei, F. Massa, B. Piccirillo, C. de Lisio, G. D. Filippis, V. Cataudella, E. Santamato, and L. Marrucci, Nat. Commun. 7, 11439 (2016).

[50] F. Caruso, A. Crespi, A. G. Ciriolo, F. Sciarrino, and R. Osellame, Nat. Commun. 7, 11682 (2016).

[51] F. Meinert, M. J. Mark, E. Kirilov, K. Lauber, P. Weinmann, M. Grobner, A. J. Daley, H.-C. Nagerl, N. Ismail, K. Wörhoff, Y. Bromberg, Y. Silberberg, M. G. Thompson, and J. L. OBrien, Science 344, 1259 (2014).

[52] C. M. Chandrashekar, R. Srikanth, and R. Laflamme, Phys. Rev. A 77, 032326 (2008).

[53] H. Majury, J. Boutari, E. O'Sullivan, A. Ferraro, and M. Paternostro, EPJ Quantum Techno. 5, 1 (2018).

[54] L. Innocenti, H. Majury, T. Giordani, N. Spagnolo, F. Sciarrino, M. Paternostro, and A. Ferraro, Phys. Rev. A 96, 062326 (2017).

[55] P. Zhang, B.-H. Liu, R.-F. Liu, H.-R. Li, F.-L. Li, and G.-C. Guo, Phys. Rev. A 81, 052322 (2010).

[56] S. K. Goyal, F. S. Roux, A. Forbes, and T. Konrad, Phys. Rev. Lett. 110, 263602 (2013).

[57] M. Brune, S. Haroche, J. M. Raimond, L. Davidovich, and N. Zagury, Phys. Rev. A 45, 5193 (1992).

[58] C. Monroe, D. M. Meekhof, B. E. King, and D. J. Wineland, Science 272, 1131 (1996).

[59] See Supplemental Material at http://link.aps.org/ supplemental/10.1103/PhysRevLett.122.020503 for further details of the experimentally generated states, scalability of the protocol, and the features that characterize SCS-based states, which includes Refs. [60,61].

[60] E. Karimi, B. Piccirillo, L. Marrucci, and E. Santamato, Opt. Lett. 34, 1225 (2009).

[61] D. F. Walls and G. J. Milburn, Quantum Optics(Springer Science \& Business Media, New York, 2012).

[62] B. Militello and A. Messina, Quantum Computing in Solid State Systems, edited by B. Ruggiero, P. Delsing, C. Granata, Y. Pashkin, and P. Silvestrini (Springer, New York, 2006).

[63] E. Schrödinger, Naturwissenschaften 23, 807 (1935).

[64] G. S. Agarwal, R. R. Puri, and R. P. Singh, Phys. Rev. A 56, 2249 (1997).

[65] W. Zhang, S. K. Goyal, F. Gao, B. C. Sanders, and C. Simon, New J. Phys. 18, 093025 (2016).

[66] V. V. Ulyanov and O. B. Zaslavskii, Group 22: Proceedings of the XXII International Colloquium on Group Theoretical Methods in Physics, edited by S. P. Corney, R. Delbourgo, and P. D. Jarvis (International Press, Cambridge, MA, 1998).

[67] R. Simon and N. Mukunda, Phys. Lett. A 143, 165 (1990).

[68] L. Marrucci, C. Manzo, and D. Paparo, Phys. Rev. Lett. 96, 163905 (2006).

[69] E. Bolduc, N. Bent, E. Santamato, E. Karimi, and R. W. Boyd, Opt. Lett. 38, 3546 (2013).

[70] V. D’Ambrosio, F. Cardano, E. Karimi, E. Nagali, E. Santamato, L. Marrucci, and F. Sciarrino, Sci. Rep. 3, 1 (2013).

[71] R. Fickler, R. Lapkiewicz, W. N. Plick, M. Krenn, C. Schaeff, S. Ramelow, and A. Zeilinger, Science 338, 640 (2012). 
[72] V. D’Ambrosio, N. Spagnolo, L. Del Re, S. Slussarenko, Y. Li, L. C. Kwek, L. Marrucci, S. P. Walborn, L. Aolita, and F. Sciarrino, Nat. Commun. 4, 2432 (2013).

[73] J. M. Radcliffe, J. Phys. A 4, 313 (1971).

[74] F. T. Arecchi, E. Courtens, R. Gilmore, and H. Thomas, Phys. Rev. A 6, 2211 (1972).

[75] D. Markham and V. Vedral, Phys. Rev. A 67, 042113 (2003).

[76] Y. L. Loh and M. Kim, Am. J. Phys. 83, 30 (2015).
[77] M. A. Nielsen and I. L. Chuang, Quantum Computantion and Quantum Information (Cambridge University Press, Cambridge, UK, 2010).

[78] T. Durt, B. G. Englert, I. Bengtsson, and K. Zyczkowski, Int. J. Quantum. Inform. 08, 535 (2010).

[79] S. Bandyopadhyay, P. O. Boykin, V. Roychowdhury, and F. Vatan, Algorithmica 34, 512 (2002).

[80] S. Brierley and S. Weigert, Phys. Rev. A 79, 052316 (2009). 